\title{
Meson Production in High Energy p+p Collisions at the RHIC Energies
}

\author{
Bao-Chun Li, Ya-Zhou Wang, and Er-Qin Wang \\ Department of Physics, Shanxi University, Taiyuan, Shanxi 030006, China \\ Correspondence should be addressed to Bao-Chun Li; libc2010@163.com
}

Received 17 April 2013; Revised 22 May 2013; Accepted 28 May 2013

Academic Editor: Fu-Hu Liu

Copyright (C) 2013 Bao-Chun Li et al. This is an open access article distributed under the Creative Commons Attribution License, which permits unrestricted use, distribution, and reproduction in any medium, provided the original work is properly cited.

\begin{abstract}
Transverse momentum spectra of mesons produced in $\mathrm{p}+\mathrm{p}$ collisions are studied in the framework of a thermalized cylinder model. In the region of high transverse momentum, the considered distributions have a tail part at the maximum energy of RHIC. A twocomponent distribution based upon the improved cylinder model is used to fit the experimental data of the PHENIX Collaboration. It is found that the improved approach can describe the meson production in the wider range of transverse momenta.
\end{abstract}

\section{Introduction}

Relativistic heavy ion collisions are performed to study nuclear matter under extreme temperature and density conditions [1]. Proton-proton collisions are conventionally used as a reference to compare with nuclear collisions and to understand the observed collective effects. And the Large Hadron Collider (LHC) [2] was originally designed to rise center-of-mass $(\mathrm{cm})$ energy to $14 \mathrm{TeV}$, which is almost 8 times the record of $1.8 \mathrm{TeV}$ achieved by the Fermilab in the United States. After the LHC data obtained in $p+p$ interactions at $\sqrt{s_{\mathrm{NN}}}=900 \mathrm{GeV}$ and $7 \mathrm{TeV}[3,4]$, the new interest in general features of $p+p$ collisions at ultrarelativistic energies appeared. In $\mathrm{p}+\mathrm{p}$ collisions, the meson spectra provide insight into the particle production mechanism and interaction in the hadronic and quark gluon plasma (QGP) phases. Furthermore, the detailed study of meson spectra is important because it acts as an ingredient for estimating the hadronic decay backgrounds in the photon, single lepton, and dilepton spectra, which are the penetrating probes of QGP. To isolate phenomena related to the dense and hot medium created in such collisions, it is also important to measure particle production in smaller collision systems like p-p and $\mathrm{d}$-Au. Measurements of transverse momentum spectra for particles produced in $p+p$ collisions are used as a baseline, to which similar measurements for heavy ion collisions are compared. In addition, the nuclear modification factor $R_{\mathrm{AA}}$ of several identified hadrons with high transfer momentum is used to probe jet quenching.

The information about the production process is retained by the final-state particle distributions in the collisions [59]. Single-hadron production at large transverse momenta in high-energy hadronic and nuclear collisions results from the fragmentation of quarks and gluons issuing from partonparton scatterings with large momentum transfer. To explain the abundant experimental data, different phenomenological mechanisms of initial coherent multiple interactions and particle transports were proposed and extended in recent years [10-15]. The comparison to model calculations can provide valuable information of the collision evolution and help better understand properties of the QGP. In particular, several theory models of high energy collisions were reported in a workshop held at the CERN Theory Institute [16]. Recently, systematic studies on the production of finalstate particles performed by the NA49 collaboration were discussed [17]. Hadronic transport models fail to describe the production of final-state particles, while the results of statistical models are generally in good agreement with the measured particle yields at available energies.

Based on the one-dimensional string model [19] and the fireball model [20], we have developed a thermalized cylinder model, which successfully describes the particle production in heavy ion collisions over an energy range from the Alternating Gradient Synchrotron (AGS) to the relativistic heavy 
ion collider (RHIC) [21-25]. In our previous work [25], the transverse momentum distributions of strange hadrons produced in $\mathrm{Cu}+\mathrm{Cu}$ and $\mathrm{Au}+\mathrm{Au}$ collisions at $\mathrm{RHIC}$ energies were explained by a single component distribution. The excitation degree of the emission source is allowed to be determined by studying the transverse momentum spectra, anisotropic flow effects, and their correlations. We found that the single component distribution only describes a narrow transverse momentum rang. Recently, the invariant differential crosssection for production of neutral mesons in $\mathrm{p}+\mathrm{p}$ collisions at $\sqrt{s_{\mathrm{NN}}}=200 \mathrm{GeV}$ were published by the PHENIX Collaboration [18]. The high $p_{T}$ reach of the transverse momentum is helpful to characterize the mechanisms of truly perturbative parton-parton scatterings and parton fragmentation in different QCD environments. Particle yields observed in these experiments inspired our work. It is interesting for us to analyze the results of pp collisions at the RHIC energies. In order to verify the thermalized cylinder model and describe the broad distribution range, in this paper, we will develop the single component spectra into a two-component distribution.

\section{The Formula}

According to the cylinder model [25], we assume the projectile and target cylinders to be formed in $\mathrm{A}+\mathrm{A}, \mathrm{d}+\mathrm{A}$ and $\mathrm{p}+\mathrm{p}$ collisions at high energies. The cylinders are wounded sources of particles. The idea comes from the observation that the process of particle production is not instantaneous, which was noted in $[26,27]$. In the reference frame where the longitudinal momentum of a produced particle vanishes, the minimal time necessary for its creation is $t_{0} \geqslant 1 / M_{T}$, where $M_{T}=$ $\sqrt{p_{T}^{2}+m^{2}}$. In the laboratory frame, the particle in question acquires some longitudinal momentum and we have

$$
t \geqslant \gamma t_{0}=\frac{E}{m_{t}^{2}}=\frac{\cosh y}{m_{t}},
$$

where $\gamma$ and $E$ are the Lorentz factor and the energy of the particle, respectively. So, the resolving power of the longitudinal distance (the uncertainty of the distance from the collision point to those at which the particle is created) is

$$
l=v t=\frac{\sinh y}{m_{t}} .
$$

When the rapidity of the produced particle is large enough so that $l>Z(b)$, where $Z(b)$ is the size of the nucleus at a given impact parameter, the particle cannot resolve individual collisions. It is natural to suggest that its creation may be insensitive to the number of collisions in the source. This is the origin of the idea of wounded sources. The concept of the wounded source may practically be applied in the whole rapidity region.

In AA collisions the cylinder is thick, and in $\mathrm{p}+\mathrm{p}$ collisions the cylinder is thin. Final-state particles are randomly produced from emission sources in the cylinder(s). The excitation degree of the side-surface region is naturally lower than that of the central axis region in the cylinders. From central axis region to side-surface region of the concerned emission source, the excitation degree is assumed to decrease linearly along the transverse axis direction [25]. When the excitation degree increases, the value of the distribution width $\sigma$ becomes bigger and bigger. So, the excitation degree can be characterized by the momentum distribution width $\sigma$. The emission points with the same excitation degree form an emission circle in the transverse momentum space. Therefore, a given $\sigma$ corresponds to wounded sources which emits a fixed density of particles. Let $\sigma_{s}$ and $\sigma_{c}$ denote the widths of the transverse momentum distribution of particles produced in the side-surface and central axis regions, respectively. The distribution of $\sigma$ can be given by

$$
f(\sigma)=C\left(\sigma_{c}-\sigma\right),
$$

with the normalization constant

$$
C=\frac{2}{\left(\sigma_{c}-\sigma_{s}\right)^{2}} .
$$

According to the single component distribution in the Monte Carlo calculation, the transverse momentum distribution of final-state particles can be given by

$$
p_{T}=\left(\sigma_{c}-\sqrt{\left(\sigma_{c}-\sigma_{s}\right)^{2}-\frac{2 R_{1}}{C}}\right) \sqrt{-2 \ln R_{2}},
$$

where $R_{1}$ and $R_{2}$ are the random variable distributed in $[0,1]$.

In our previous work [25], transverse momentum spectra of strange particles produced in high-energy collisions were investigated by the above thermalized cylinder model. Considering the simply cylinder shape, we obtained the emission source location dependence of the exciting degree specifically. It is shown that the single component distribution, that is, (5), is successful in describing the experimental results measured by the STAR and PHOBOS Collaborations. Moreover, we found that the single component distribution can describe a narrow transverse momentum range. To explain the wider transverse momentum spectra of identified particles produced in $\mathrm{p}+\mathrm{p}$ collisions, we need to consider the relative importance of hard versus soft processes in the particle production mechanisms at different energies. Hard partonparton scatterings with large momentum transfer occur on a short time scale and are governed by perturbative QCD. The bulk of particle production occurs via soft processes (with low momentum transfer and consequently longer time scales) which are described with phenomenological models.

In order to describe the invariant cross-section of hadrons as a function of $p_{T}$ over a wide range, Hagedorn proposed an empirical formula [28], which is described as "inspired by QCD” and is given by

$$
\begin{aligned}
E \frac{d^{3} N}{d p^{3}} & =\frac{B}{\left(1+p_{T} / \lambda\right)^{n}} \\
& \simeq B \begin{cases}\left(\frac{-n p_{T}}{\lambda}\right) & \text { for } p_{T} \longrightarrow 0, \\
\left(\frac{\lambda}{p_{T}}\right)^{n} & \text { for } p_{T} \longrightarrow \infty,\end{cases}
\end{aligned}
$$


where $B, \lambda$, and $n$ are fit parameters. The limiting cases show that the distribution is an exponential form at low transverse momenta, but it is a power law at large transverse momenta, which came from "QCD inspired" quark interchange model [29],

$$
E \frac{d^{3} N}{d p^{3}} \sim\left(m_{T}^{2}\right)^{-4} \sim \frac{1}{\left(p_{T}\right)^{8}} .
$$

The dominant contribution to the inclusive cross section is low $p_{T}$ particles. However, with center of mass energy increasing, the hardening of the $p_{T}$ distribution implies an increase of the transverse momentum. At both low $p_{T}$ and high $p_{T}$, UAl collaboration [30] gives a hybrid form,

$$
\begin{array}{rlrl}
E \frac{d^{3} N}{d p^{3}} & =A \exp \left(-b m_{T}\right), \quad & \text { for } p_{T}<p_{T}^{c} \\
& =\frac{B}{\left(1+p_{T} / p_{0}\right)^{n}}, \quad \text { for } p_{T}>p_{T}^{c}
\end{array}
$$

where $p_{T}^{c}$ is a free parameter. In order to give a good description of pion spectra in wide $p_{T}$ range, the PHENIX Collaboration $[18,31,32]$ found a single form referred as the modified Hagedorn formula. The modification is to better describe the $\pi^{0}$ spectrum for wider $p_{T}$ range, in particular at high $p_{T}$ where the spectrum behaves close to a simple power law function. The single formula has been used to successfully describe the hadron spectra measured in $\mathrm{p}$ - $\mathrm{p}$ collisions at different energies $[18,31,32]$. The formula has been extensively used in terms of $m_{T}$,

$$
E \frac{d^{3} N}{d p^{3}}=\frac{B}{\left[\exp \left(-a m_{T}-b m_{T}^{2}+m_{T} / \lambda\right)\right]^{n}},
$$

where $a$ and $b$ are the fit parameters. The distribution function is close to an exponential form at low $p_{T}$ and a pure power law form at high $p_{T}$. Similarly, our model should be improved to a two-component distribution,

$$
\begin{aligned}
P_{T}= & k\left(\sigma_{1 c}-\sqrt{\left(\sigma_{1 c}-\sigma_{1 s}\right)^{2}-2 R_{1} / C_{1}}\right) \sqrt{-2 \ln R_{2}} \\
& +(1-k)\left(\sigma_{2 c}-\sqrt{\left(\sigma_{2 c}-\sigma_{2 s}\right)^{2}-2 R_{3} / C_{2}}\right) \sqrt{-2 \ln R_{4}}
\end{aligned}
$$

where $R_{3}$ and $R_{4}$ are the random variable distributed in $[0,1], k(1-k)$ and $C_{1}\left(C_{2}\right)$ are the contribution coefficient and normalization constant of the first (second) component respectively, and $\sigma_{1 s}\left(\sigma_{2 s}\right)$ and $\sigma_{1 c}\left(\sigma_{2 c}\right)$ are the momentum distribution widths of particles produced in side-surface region and central axis region of the first (second) component, respectively. Our calculation shows that $C_{1} \neq C_{2}$. The first and second items in (10) correspond to the contributions of soft production and hard emission, respectively.

The basic model assumes the cylinders with the emitted momentum distribution width changing from the side-surface to the central axis. In fact, except for the most central collisions, the active (overlap) region is certainly not cylindrical.
We could understand the "cylinder" as a nonperfect cylinder along the beam direction in the momentum space but not a perfect cylinder in the coordinate space. The emission sources with the same excitation degree stay at the same (sub)surface. This situation is similar to the equipotential surface in electromagnetism.

\section{Comparison with PHENIX Results}

The invariant differential cross-sections of neutral mesons produced in $\mathrm{p}$ - $\mathrm{p}$ collisions at nucleon-nucleon center-ofmass energy $\sqrt{s_{\mathrm{NN}}}=200 \mathrm{GeV}$ in various decay modes are presented in Figure 1. The symbols represent the experimental data of the PHENIX Collaboration [18] and the curves are our calculated results by using (10). The $\chi^{2}$ testing provides statistical indication of the most probable value of corresponding parameters. The values of $k, \sigma_{1 c}-\sigma_{1 s}$, and $\sigma_{2 c}-\sigma_{2 s}$ obtained by fitting the experimental data are given in Table 1 with $\chi^{2}$ per degree of freedom (dof). The maximum value of the observed $p_{T}$ reaches about $13.0 \mathrm{GeV}$. We see that the calculated results approximately agree with the experimental data for neutral mesons in the region. The values of $k, \sigma_{1 c}$, and $\sigma_{2 c}$ are taken to be $99.98 \pm 0.02,1.70 \pm 0.12$ and $4.64 \pm 0.35$, respectively. Both $\sigma_{1 s}$, and $\sigma_{2 s}$ values do not change obviously.

To compare these results to other particles, we show the invariant differential cross sections of different particles produced in $\mathrm{p}+\mathrm{p}$ collisions at $\sqrt{s_{\mathrm{NN}}}=200 \mathrm{GeV}$ in various decay modes in Figure 2. The different symbols are the experimental data of the PHENIX Collaboration [18]. The solid curves are our results calculated by the model. By fitting the experimental data, the parameter values are given in Table 1 with the values of $\chi^{2} /$ dof. Similar to Figure 1 , the values of $\sigma_{1 s}\left(\sigma_{2 s}\right)$ do not change significantly. We see again that the model describes approximately the invariant differential cross-sections of final-state mesons produced in $\mathrm{p}+\mathrm{p}$ collisions at the highest RHIC energy. Compared with the case of Figure 1, the values of parameters do not change significantly.

In Figure 3, we present the invariant cross section at midrapidity for $\eta$ in $\mathrm{p}+\mathrm{p}$ collisions at $\sqrt{s_{\mathrm{NN}}}=200 \mathrm{GeV}$. The symbols are the experimental data of the PHENIX Collaboration $[33,34]$. The solid curves are our results calculated by the model. The dotted and dashed curves are our results corresponding to the contributions of the first and second items in (10). The parameter values and corresponding $\chi^{2} /$ dof are given in Table 1 . One can see the particle production of soft production and hard emission intuitively in the figure.

In order to testify the validity of the model, Figure 4 shows the invariant $\pi^{0}$ cross section, $E d^{3} \sigma / d^{3} p$, in $\mathrm{p}+\mathrm{p}$ collisions at different RHIC energies. The symbols are the experimental data of the PHENIX Collaboration [35]. The solid curves are our results calculated by the model. The dotted and dashed curves are our results corresponding to the contributions of the first and second items in (10). The values of $k, \sigma_{1 s} \sim \sigma_{1 c}$, and $\sigma_{2 s} \sim \sigma_{2 c}$ are given in Table 2 with the corresponding $\chi^{2} / \mathrm{dof}$. The calculated results are in agreement with the experimental data in $\mathrm{p}$-p collisions at the RHIC energies. The $k$ values decrease with the increase of 
TABLE 1: Values of the parameters $k, \sigma_{1 s} \sim \sigma_{1 c}$, and $\sigma_{2 s} \sim \sigma_{2 c}$ obtained by fitting the experimental $p_{T}$ spectra in Figures 1, 2, and 3. The units of $\sigma_{1 s}, \sigma_{1 c}, \sigma_{2 s}$, and $\sigma_{2 c}$ are $\mathrm{GeV} / \mathrm{c}$.

\begin{tabular}{lcccc}
\hline Particle & $k(\%)$ & $\sigma_{1 s} \sim \sigma_{1 c}(\times 0.1)$ & $\sigma_{2 s} \sim \sigma_{2 c}(\times 0.1)$ & $\chi^{2} / \mathrm{dof}$ \\
\hline$\omega$ & $99.97 \pm 0.01$ & $0.09 \pm 0.02 \sim 1.66 \pm 0.10$ & $1.28 \pm 0.10 \sim 4.55 \pm 0.24$ & 1.000 \\
$K$ & $99.97 \pm 0.01$ & $0.06 \pm 0.01 \sim 1.60 \pm 0.12$ & $1.22 \pm 0.10 \sim 4.50 \pm 0.21$ & 1.310 \\
$\eta^{\prime}$ & $99.98 \pm 0.01$ & $0.07 \pm 0.01 \sim 1.68 \pm 0.10$ & $1.28 \pm 0.10 \sim 4.65 \pm 0.27$ & 0.472 \\
$\phi$ & $99.99 \pm 0.00$ & $0.05 \pm 0.01 \sim 1.72 \pm 0.10$ & $1.30 \pm 0.10 \sim 4.75 \pm 0.24$ & 0.407 \\
$\pi$ & $99.96 \pm 0.01$ & $0.08 \pm 0.01 \sim 1.65 \pm 0.10$ & $1.26 \pm 0.10 \sim 4.52 \pm 0.22$ & 0.210 \\
$\eta$ & $99.97 \pm 0.01$ & $0.06 \pm 0.01 \sim 1.61 \pm 0.11$ & $-24 \pm 0.11 \sim 4.48 \pm 0.26$ & 0.240 \\
$p+\bar{p}$ & $100.00 \pm 0.01$ & $0.10 \pm 0.02 \sim 1.78 \pm 0.10$ & - & 0.278 \\
$J / \psi$ & $100.00 \pm 0.01$ & $0.16 \pm 0.03 \sim 2.25 \pm 0.15$ & - & 0.447 \\
$\psi^{\prime}$ & $100.00 \pm 0.01$ & $0.18 \pm 0.04 \sim 2.10 \pm 0.12$ & \\
\hline
\end{tabular}

TABLE 2: Values of the parameters $k, \sigma_{1 s} \sim \sigma_{1 c}$, and $\sigma_{2 s} \sim \sigma_{2 c}$ obtained by fitting the experimental $p_{T}$ spectra Figure 4 . The units of $\sigma_{1 s}, \sigma_{1 c}$, $\sigma_{2 s}$, and $\sigma_{2 c}$ are $\mathrm{GeV} / \mathrm{c}$.

\begin{tabular}{cccccc}
\hline Particle & Energy $(\mathrm{GeV})$ & $k(\%)$ & $\sigma_{1 s} \sim \sigma_{1 c}(\times 0.1)$ & $\sigma_{2 s} \sim \sigma_{2 c}(\times 0.1)$ & - \\
$\pi^{0}$ & 22.4 & $100.00 \pm 0.01$ & $0.05 \pm 0.01 \sim 1.05 \pm 0.06$ & & -600 \\
& 62.4 & $99.95 \pm 0.01$ & $0.05 \pm 0.01 \sim 1.26 \pm 0.09$ & $1.10 \pm 0.07 \sim 4.14 \pm 0.20$ \\
& 200 & $99.69 \pm 0.02$ & $0.07 \pm 0.01 \sim 1.69 \pm 0.12$ & $1.29 \pm 0.10 \sim 4.58 \pm 0.25$ & 0.422 \\
\hline
\end{tabular}

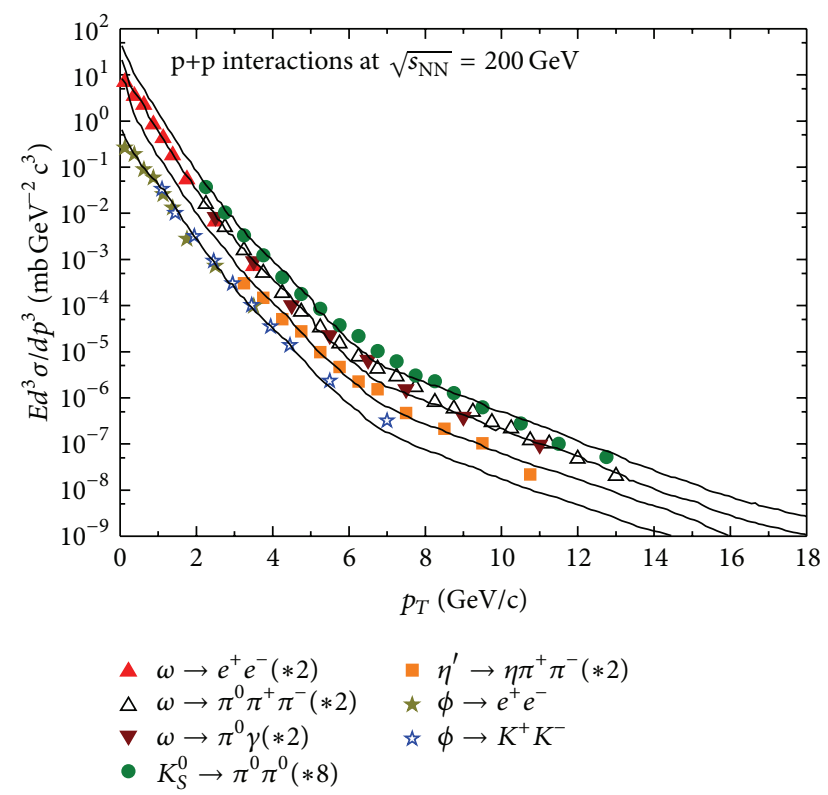

FIGURE 1: Invariant differential cross-section of neutral mesons measured in $\mathrm{p}+\mathrm{p}$ collisions at $\sqrt{s_{\mathrm{NN}}}=200 \mathrm{GeV}$ in various decay modes. Experimental data measured by the PHENIX Collaboration [18] are shown by the scattered symbols. Our results calculated from the cylinder model are shown by the curves.

the collision energy, and $\sigma_{s}$ has no obvious change, equals only about $0.60 \mathrm{GeV} / \mathrm{c}$. The values of $\sigma_{1 c}(0.99-1.81 \mathrm{GeV} / \mathrm{c})$ and $\sigma_{2 c}(3.94-4.83 \mathrm{GeV} / \mathrm{c})$ increase with the collision energy increasing. As discussed in detail in [25], the excitation degree of emission source on the cylinder central axis becomes higher due to the higher collision energy.

We would like to point out that the calculated results in Figures 1-4 are obtained by (10), which is a general

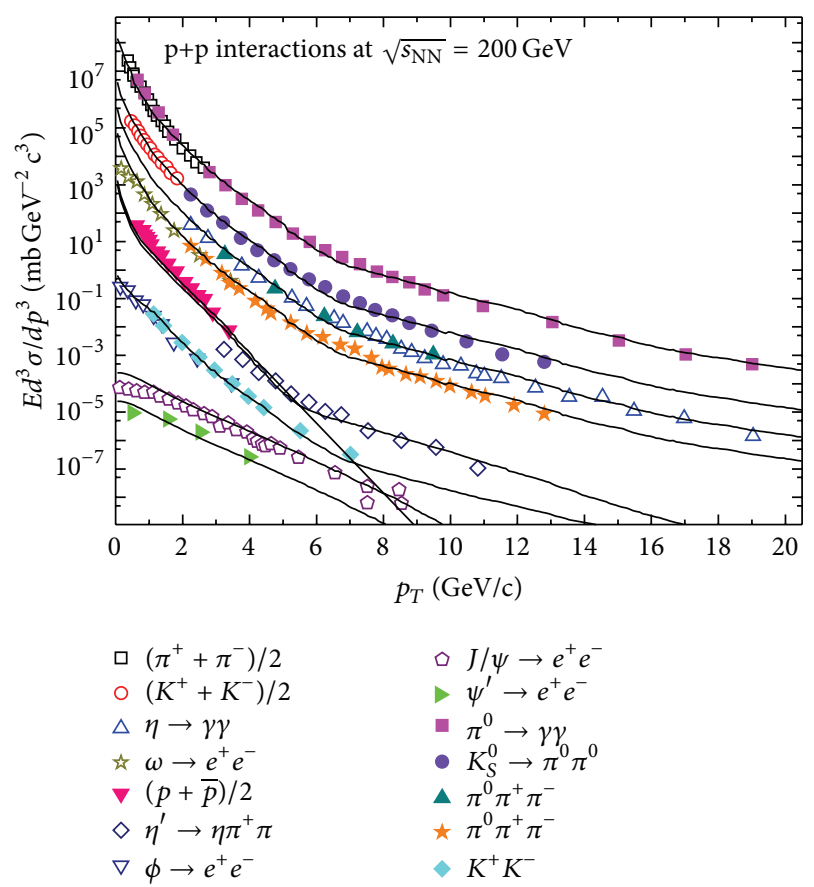

FIGURE 2: Invariant differential cross-sections of different particles measured in $\mathrm{p}+\mathrm{p}$ collisions at $\sqrt{s_{\mathrm{NN}}}=200 \mathrm{GeV}$ in various decay modes. Experimental data measured by the PHENIX Collaboration [18] are shown by the scattered symbols. Our results calculated from the cylinder model are shown by the curves.

representation in the Monte Carlo method. Equations (6)(9) are only used to explain why the two components need to be considered in (10). The curves in the figures are numerical results from (10). Some of them are not smooth due to low statistics. 


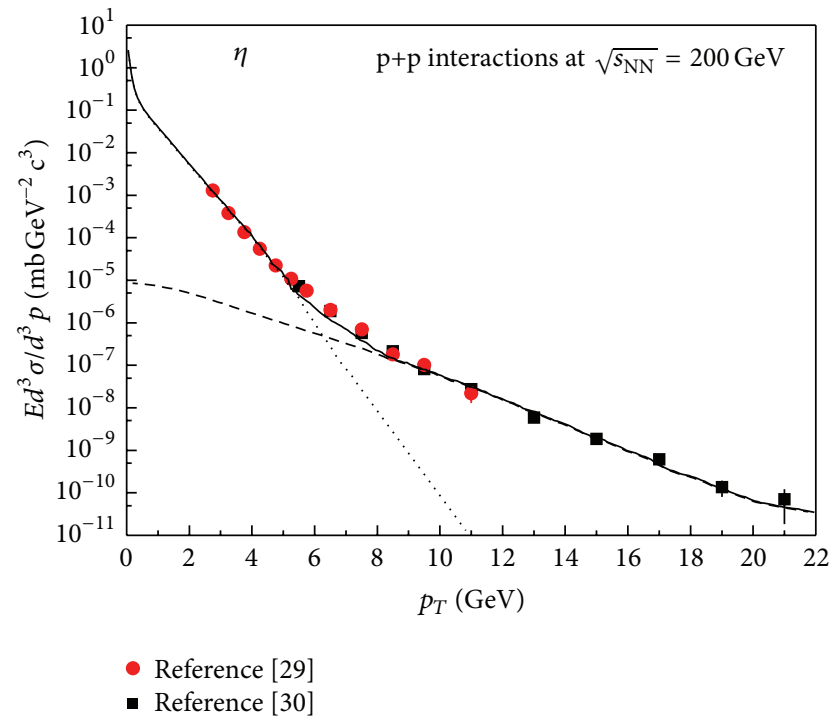

FIgURE 3: Invariant $\eta$ cross section at midrapidity as a function of transverse momentum in $\mathrm{p}+\mathrm{p}$ at $\sqrt{s_{\mathrm{NN}}}=200 \mathrm{GeV}$. The error bars are the statistical uncertainties. Experimental data taken from the PHENIX Collaboration $[33,34]$ are shown by the scattered symbols. Our results calculated from the cylinder model are shown by the curves.

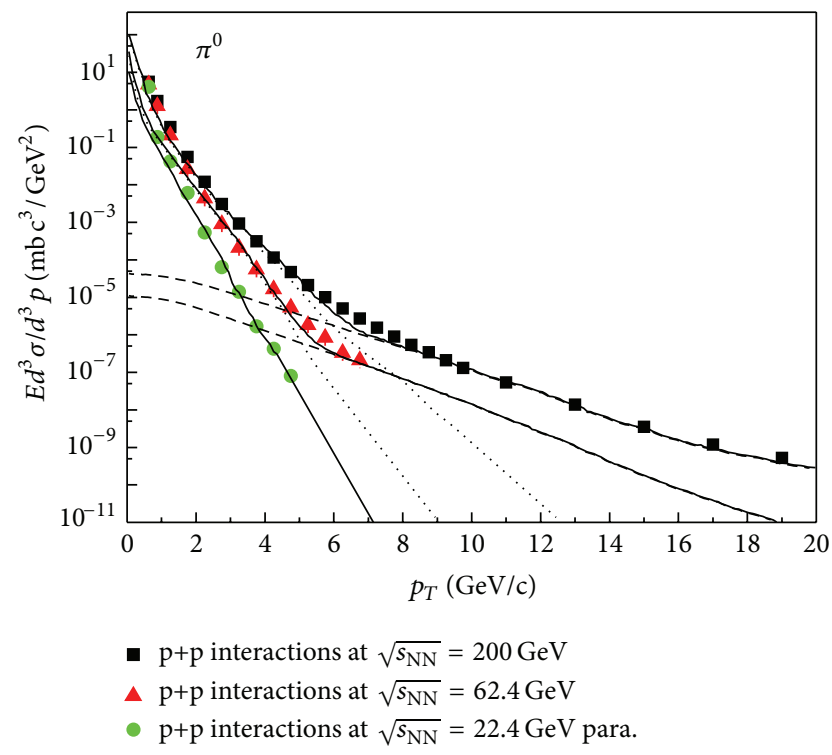

FIGURE 4: Invariant $\pi^{0}$ cross-section as a function of transverse momentum in $\mathrm{p}+\mathrm{p}$ collisions at different RHIC energies. The error bars are the quadratic sum of statistical and systematic uncertainties. Experimental data taken from the PHENIX Collaboration [35] are shown by the scattered symbols. Our results calculated from the cylinder model are shown by the curves.

\section{Discussion}

The above comparison shows that the improved cylinder model can be used in the description of the meson production in the wider range of transverse momenta. The values of $\chi^{2} /$ dof for all fits are shown in Tables 1 and 2 . The maximum value is 1.420 , and the minimum value is 0.246 . We demonstrate that the calculated results are in good agreement with the available experimental data. In our calculation, $k$ is the most important parameter which determines the distributive slope and range. In most cases, the second item cannot be neglected due to the contribution of the hard process. A large $k(\simeq 1)$ gives a precipitous and narrow distribution, whereas a smaller $k(<1)$ gives a subdued and wide distribution. The second item in $(10)$ is very small $(<0.01)$ and does not contribute to the spectra at the lowest RHIC energy. In $p+p$ collisions for different energies, we see that $1-k$ increases slightly with the increasing of collision energies. This means that the contribution of hard emission increases slightly. For the asymmetric system of $\mathrm{d}+\mathrm{Au}$ collisions, it is predicted that the values of $k$ do not change significantly due to the spatial asymmetry of the collision nucleons. Scattering processes at high energy hadron colliders can be classified as either hard or soft. Quantum Chromodynamics (QCD) is the underlying theory for all such processes, but, the approach and level of understanding arevery different for the two cases. For hard processes (e.g., Higgs boson or high $p_{T}$ jet production), the rates and event properties can be predicted by using perturbation theory. In addition, for soft processes (e.g., the total cross section, the underlying event, etc.), the rates and properties are dominated by nonperturbative QCD effects, which are less well understood. For many hard processes, soft interactions are occurring along with the hard interactions and their effects must be understood for comparisons to be made to perturbative predictions. An understanding of the rates and characteristics of predictions for hard processes, both signals and backgrounds, using perturbative QCD (pQCD) is crucial for both the Tevatron and LHC. We are in a position to evaluate the soft and hard contributions to the observed spectra by using $k$ in the statistical model.

The particles produced in high-energy nuclear collisions have attracted much attention since people are trying to understand the properties of strongly interacting quarkgluon plasma by studying the possible production mechanisms [36, 37]. The final-state particles are emitted isotropically in the rest frame of emission sources with the different excitation degree in collisions. If we consider that the local emission source has a motion in the transverse direction, there are interactions among the emission sources, then the transverse flow (directed flow and elliptic flow) can be explained by the model. Thermal-statistical models have been successful in describing particle yields in various systems at different energies. The cylinder model is developed from the fireball model, which is suggested in heavy-ion collisions [20]. The excitation degree varies with location in the cylinder. In the previous work [25], we obtained the emission source location dependence of the exciting degree specifically. From central axis to side-surface of the cylinder, the excitation degree of the emission source decreases linearly with the direction of radius. The excitation degree can easily be characterized by the corresponding distribution width, which is written as (4) by using the Monte Carlo calculation. In the model, because of the influence of the emission source temperature, the values of $\sigma_{s}$ are much smaller than those 
of the $\sigma_{c}$. It is consist with the conclusion of [15], where the temperature of the fireball decrease linearly with time.

In the present work, we improve the method by considering the difference between the soft and hard emission. The parameters $\sigma_{1 c}\left(\sigma_{1 s}\right)$ and $\sigma_{2 c}\left(\sigma_{2 s}\right)$ are used to describe the excitation degrees of emission sources closing to the sidesurface and central axis of the cylinders, respectively. They are in fact reflections of the excitation degree of the soft emission process and the hard emission process, respectively. Our results agree well with the considered distributions of mesons with high transverse momenta produced in nuclear collisions at RHIC energies. At finite temperature, the stronger the collision strength, the larger the excitation degree. Therefore, on the central axis of the cylinder, the interaction between particles is strongest, and the excitation degree is highest. For different collision energies, our results in the improved cylinder model show that the parameters $\sigma_{1 c}$ and $\sigma_{2 c}$ slowly increase with the increasing of energy, and no obvious change can be observed in the values of $\sigma_{s 1}\left(\sigma_{s 2}\right)$. The dependence of parameters on energies renders that intranuclear cascade collisions in the central axis regions play a more important role at higher energy. In the side-surface regions, a high multiplicity at high energy does not contribute to a high excitation degree due to weak intranuclear cascade collisions. In our opinion, high $p_{T}$ hadrons come primarily from the central region due to their large emission angles. And high momentum hadrons come primarily from the side-surface region due to their weak cascade collisions.

\section{Conclusions}

In summary, the transverse momentum distributions of mesons produced in $\mathrm{p}+\mathrm{p}$ collisions at the RHIC energies have been studied in the framework of the improved cylinder model. The model is successful in description of the high transverse momentum meson production. Based on our phenomenological approach, we evaluated the soft and hard contributions to the observed spectra. At the same time, it can offer some information about soft and hard interactions in the collisions. In our previous work, a rudimentary investigation of the azimuthal anisotropy has been carried basing upon the cylinder model. Combined with the present work, the model can be used to describe uniformly the momentum distributions of final-state particles produced in high energy collisions.

\section{Acknowledgments}

This work is supported by the National Natural Science Foundation of China under Grant no. 11247250, no. 11005071, and no. 10975095, the National Fundamental Fund of Personnel Training under Grant no. J1103210, the Shanxi Provincial Natural Science Foundation under Grant no. 2013021006 and no. 2011011001, the Open Research Subject of the Chinese Academy of Sciences Large-Scale Scientific Facility under Grant no. 2060205, and the Shanxi Scholarship Council of China.

\section{References}

[1] H. Caines, "Recent results from RHIC: first steps towards quantitative QGP measurements," AIP Conference Proceedings, vol. 1422, p. 5, 2012.

[2] R. Aaij, C. Abellan Beteta, A. Adametz, B. Adeva, M. Adinolfi, $\mathrm{LHCb}$ Collaboration et al., "Measurement of prompt hadron production ratios in $p p$ collisions at $\sqrt{ } s=0.9$ and $7 \mathrm{TeV}$," European Physical Journal C, vol. 72, p. 2168, 2012.

[3] K. Aamodt, N. Abel, U. Abeysekara et al., "Transverse momentum spectra of charged particles in proton-proton collisions at $\sqrt{s}=900 \mathrm{GeV}$ with ALICE at the LHC," Physics Letters B, vol. 693, no. 2, pp. 53-68, 2010.

[4] V. Khachatryan, A. M. Sirunyan, A. Tumasyan et al., "Transverse-momentum and pseudorapidity distributions of charged hadrons in pp collisions at $\sqrt{ } s=7 \mathrm{TeV}$," Physical Review Letters, vol. 105, Article ID 022002, 14 pages, 2010.

[5] B. B. Back, M. D. Baker, M. Ballintijn et al., "Centrality dependence of charged hadron transverse momentum spectra in $\mathrm{Au}+\mathrm{Au}$ collisions from $\sqrt{ } s_{N N}=62.4$ to $200 \mathrm{GeV}$," Physical Review Letters, vol. 94, Article ID 082304, 4 pages, 2005.

[6] B. B. Back, M. D. Baker, D. S. Barton et al., "Charged hadron transverse momentum distributions in $\mathrm{Au}+\mathrm{Au}$ collisions at $\sqrt{s_{N N}}=200$ GeV," Physics Letters B, vol. 578, pp. 297-303, 2004.

[7] J. Adams, M. M. Aggarwal, Z. Ahammed et al., "Scaling properties of hyperon production in $\mathrm{Au}+\mathrm{Au}$ collisions at $\sqrt{s_{N N}}=$ 200 GeV," Physical Review Letters, vol. 98, Article ID 062301, 6 pages, 2007.

[8] G. M. S. Vasconcelos and STAR Collaboration, "Multi-strange particle production in relativistic heavy ion collisions at $\sqrt{s_{N N}}=$ 62.4 GeV," Journal of Physics G, vol. 37, Article ID 094034, 2010.

[9] R. Preghenella and ALICE Collaboration, "Measurement of the production cross section for $\mathrm{Z} /$ gamma $^{*}$ in association with jets in $p p$ collisions at $\sqrt{s}=7 \mathrm{TeV}$ with the ATLAS detector," Physical Review D, vol. 85, Article ID 032009, 2012.

[10] M. L. Miller, K. Reygers, S. J. Sanders, and P. Steinberg, "Glauber modeling in high-energy nuclear collisions," Annual Review of Nuclear and Particle Science, vol. 57, pp. 205-243, 2007.

[11] H. De Vries, C. W. De Jager, and C. De Vries, "Nuclear chargedensity-distribution parameters from elastic electron scattering," Atomic Data and Nuclear Data Tables, vol. 36, no. 3, pp. 495-536, 1987.

[12] P. Braun-Munzinger, D. Magestro, K. Redlich, and J. Stachel, "Hadron production in Au-Au collisions at RHIC," Physics Letters B, vol. 518, no. 1-2, pp. 41-46, 2001.

[13] J. Rafelski and J. Letessier, "Testing limits of statistical hadronization," Nuclear Physics A, vol. 715, pp. 98c-107c, 2003.

[14] V. Roy and A. K. Chaudhuri, "Transverse momentum spectra and elliptic flow in ideal hydrodynamics and geometric scaling," Physical Review C, vol. 81, Article ID 067901, 4 pages, 2010.

[15] T. Song, C. M. Ko, S. H. Lee, and J. Xu, "J/ $\Psi$ production and elliptic flow in relativistic heavy-ion collisions," Physical Review C, vol. 83, Article ID 014914, 13 pages, 2011.

[16] N. Armesto, N. Borghini, S. Jeon, U. A. Wiedemann, S. Abreu, S. V. Akkelin et al., "Heavy-ion collisions at the LHC-Last call for predictions," Journal of Physics G, vol. 35, Article ID 054001, 2008.

[17] C. Blume and NA49 Collaboration, "Strangeness production at the SPS," Nonlinear Phenomena in Complex Systems, vol. 12, p. $385,2009$. 
[18] A. Adare, S. Afanasiev, C. Aidala et al., "Measurement of neutral mesons in $\mathrm{p}+\mathrm{p}$ collisions at $\sqrt{s}=200 \mathrm{GeV}$ and scaling properties of hadron production," Physical Review D, vol. 83, Article ID 052004, 2011.

[19] K. Werner, "Strings, pomerons and the VENUS model of hadronic interactions at ultrarelativistic energies," Physics Reports, vol. 232, no. 2-5, pp. 87-299, 1993.

[20] G. D. Westfall, J. Gosset, P. J. Johansen, A. M. Poskanzer, and W. G. Meyer, "Nuclear fireball model for proton inclusive spectra from relativistic heavy-ion collisions," Physical Review Letters, vol. 37, pp. 1202-1205, 1976.

[21] F. H. Liu and Y. A. Panebratsev, "Baryon rapidity distribution in nucleus-nucleus collisions at ultrarelativistic energies," Physical Review C, vol. 59, pp. 1193-1195, 1999.

[22] F. H. Liu and Y. A. Panebratsev, "Pseudorapidity distribution of shower particles in heavy ion induced interactions in nuclear emulsion at high energy," Physical Review C, vol. 59, no. 3, pp. 1798-1801, 1999.

[23] F. H. Liu, "Particle production in $\mathrm{Au}-\mathrm{Au}$ collisions at RHIC energies," Physics Letters B, vol. 583, no. 1-2, pp. 68-72, 2004.

[24] F. H. Liu, Y. Yuan, and M. Y. Duan, "Widths of charged particle pseudorapidity density distributions in high energy $p \bar{p}$ and $A A$ collisions," Nuclear Physics A, vol. 801, no. 3-4, pp. 154-168, 2008.

[25] B. C. Li, Y. Y. Fu, L. L. Wang, E. Q. Wang, and F. H. Liu, "Transverse momentum distributions of strange hadrons produced in nucleus-nucleus collisions at $\sqrt{s_{N N}}=62.4$ and $200 \mathrm{GeV}$," Journal of Physics G, vol. 39, Article ID 025009, 2012.

[26] L. Landau and I. Pomeranchuk, "Limits of applicability of the theory of bremsstrahlung electrons and pair production at highenergies," Doklady Akademii Nauk, vol. 92, pp. 535-536, 1953.

[27] L. Landau and I. Pomeranchuk, "Electron cascade process at very high-energies," Doklady Akademii Nauk, vol. 92, pp. 735$738,1953$.

[28] R. Hagedorn, "Multiplicities, $\mathrm{P}(\mathrm{t})$ distributions and the expected hadron," Rivista Del Nuovo Cimento, vol. 6N10, pp. 1-50, 1984.

[29] R. Balnkenbecler and S. J. Brodsky, "Unified description of inclusive and exclusive reactions at all momentum transfers," Physical Review D, vol. 10, no. 9, pp. 2973-2992, 1974.

[30] C. Albajar, M. G. Albrow, O. C. Allkofer et al., "A study of the general characteristics of proton-antiproton collisions at $\sqrt{s}=$ 0.2 to 0.9 TeV," Nuclear Physics B, vol. 335, p. 261, 1990.

[31] A. Afanasiev, S. Adare, C. Aidala et al., "Detailed measurement of the e+e- pair continuum in $\mathrm{p}+\mathrm{p}$ and $\mathrm{Au}+\mathrm{Au}$ collisions at $\sqrt{s}=200 \mathrm{GeV}$ and implications for direct photonproduction," Physical Review C, vol. 81, Article ID 034911, 2010.

[32] A. Adare, PHENIX Collaboration et al., "Heavy-quark production in $\mathrm{p}+\mathrm{p}$ and energy loss and flow of heavy quarks in $\mathrm{Au}+\mathrm{Au}$ collisions at $\sqrt{s}=200 \mathrm{GeV}$," Physical Review C, vol. 84, Article ID 044905, 2011.

[33] S. S. Adler, S. Afanasiev, C. Aidala et al., "Centrality dependence of $\pi^{0}$ and $\eta$ production at large transverse momentum in $\sqrt{s}=200 \mathrm{GeV} d+$ Au collisions," Physical Review Letters, vol. 98, Article ID 172302, 2007.

[34] A. Adare, S. Afanasiev, C. Aidala et al., “Transverse momentum dependence of $\eta$ meson suppression in $\mathrm{Au}+\mathrm{Au}$ collisions at $\sqrt{s_{N N}}=200$ GeV," Physical Review C, vol. 82, Article ID 011902, 7 pages, 2010.

[35] A. Adare, S. Afanasiev, C. Aidala et al., "Onset of $\pi^{0}$ Suppression Studied in $\mathrm{Cu}+\mathrm{Cu}$ Collisions at $\sqrt{s}_{N N}=22.4,62.4$, and 200 GeV," Physical Review Letters, vol. 101, Article ID 162301, 2008.
[36] A. Andronic, P. Braun-Munzinger, and J. Stachel, "Hadron production in central nucleus-nucleus collisions at chemical freezeout," Nuclear Physics A, vol. 772, no. 3-4, pp. 167-199, 2006.

[37] A. Andronic, P. Braun-Munzinger, and J. Stachel, "Thermal hadron production in relativistic nuclear collisions," Acta Physica Polonica B, vol. 40, no. 4, pp. 1005-1012, 2009. 

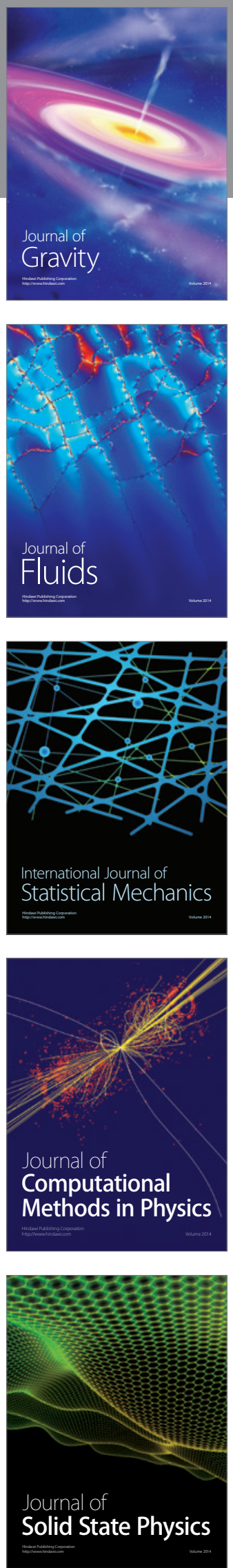

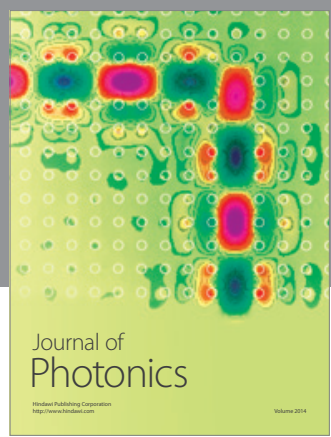

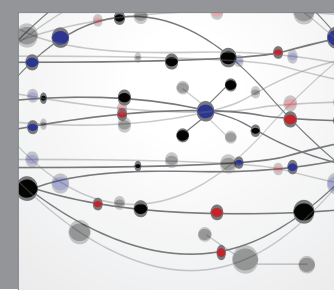

The Scientific World Journal

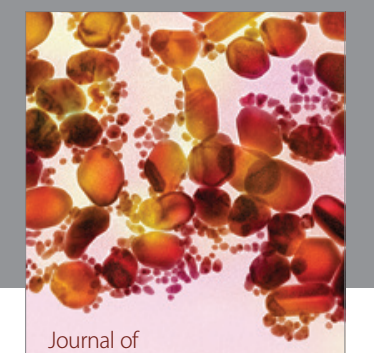

Soft Matter
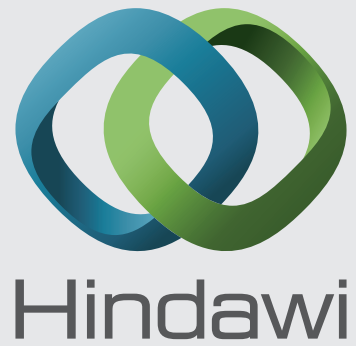

Submit your manuscripts at

http://www.hindawi.com
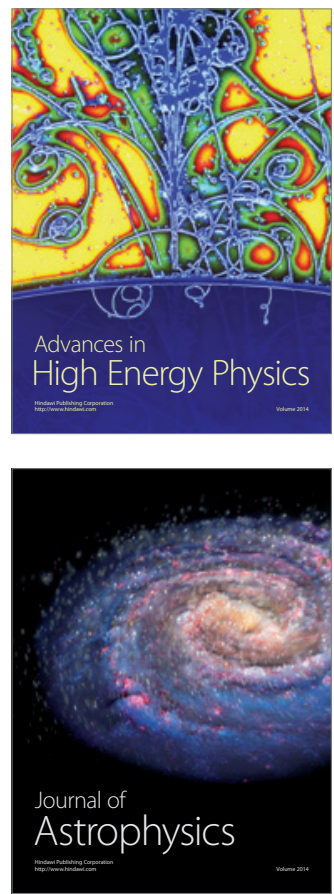
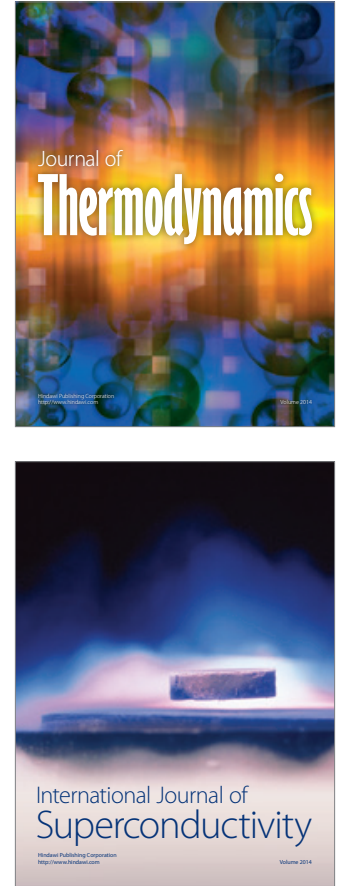
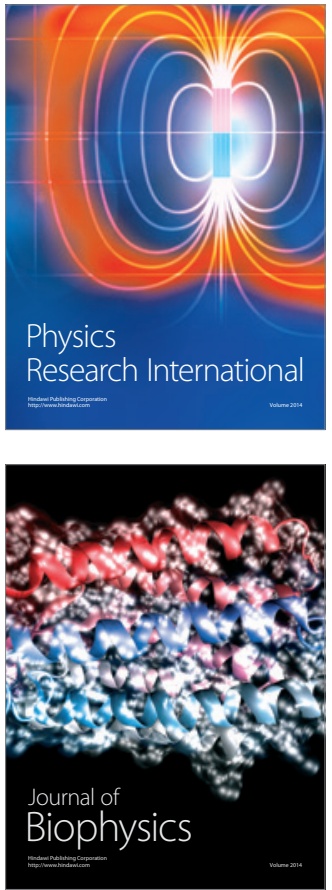
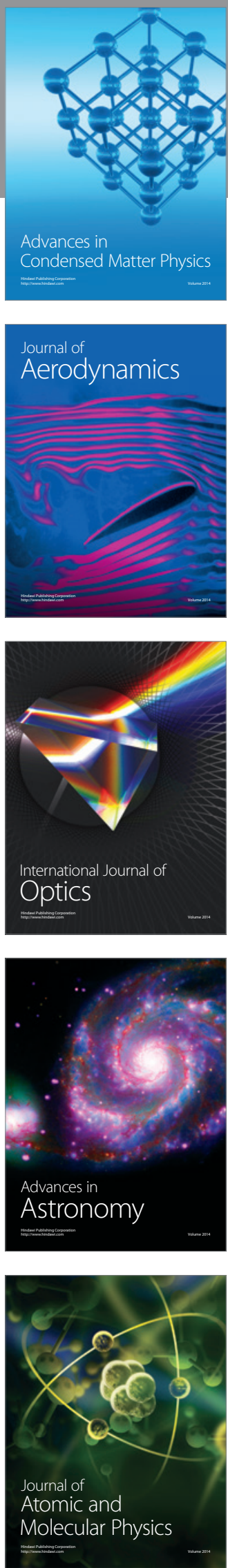